\title{
TOEPLITZ BAND MATRICES WITH EXPONENTIALLY GROWING CONDITION NUMBERS*
}

\author{
A. BÖTTCHER ${ }^{\dagger}$ AND S. GRUDSKY $\ddagger$
}

\begin{abstract}
The paper deals with the spectral condition numbers $\kappa\left(T_{n}(b)\right)$ of sequences of Toeplitz matrices $T_{n}(b)=\left(b_{j-k}\right)_{j, k=1}^{n}$ as $n$ goes to infinity. The function $b\left(e^{i \theta}\right)=\sum_{k} b_{k} e^{i k \theta}$ is referred to as the symbol of the sequence $\left\{T_{n}(b)\right\}$. It is well known that $\kappa\left(T_{n}(b)\right)$ may increase exponentially if the symbol $b$ has very strong zeros on the unit circle $\mathbb{T}=\{z \in \mathbb{C}:|z|=1\}$, for example, if $b$ vanishes on some subarc of $\mathbb{T}$. If $b$ is a trigonometric polynomial, in which case the matrices $T_{n}(b)$ are band matrices, then $b$ cannot have strong zeros unless it vanishes identically. It is shown that the condition numbers $\kappa\left(T_{n}(b)\right)$ may nevertheless grow exponentially or even faster to infinity. In particular, it is proved that this always happens if $b$ is a trigonometric polynomial which has no zeros on $\mathbb{T}$ but nonzero winding number about the origin.

The techniques employed in this paper are also applicable to Toeplitz matrices generated by rational symbols $b$ and to the condition numbers associated with $l^{p}$ norms $(1 \leq p \leq \infty)$ instead of the $l^{2}$ norm.
\end{abstract}

Key words. Toeplitz matrix, band matrix, matrix norm, condition number

AMS subject classifications. 15A12, 15A60, 47B35, 65F35

1. Introduction and main results. Let $l_{n}^{p}(1 \leq p \leq \infty)$ be the linear space $\mathbb{C}^{n}$ with the $l^{p}$ norm,

$$
\|x\|_{p}=\left(\sum_{j}\left|x_{j}\right|^{p}\right)^{1 / p} \text { for } 1 \leq p<\infty, \quad\|x\|_{\infty}=\max _{j}\left|x_{j}\right| .
$$

Every $n \times n$ matrix $A_{n}$ induces an operator on $l_{n}^{p}$, and we let $\left\|A_{n}\right\|_{p}$ stand for the norm of this operator,

$$
\left\|A_{n}\right\|_{p}=\sup _{x \neq 0}\left\|A_{n} x\right\|_{p} /\|x\|_{p}
$$

The condition number $\kappa_{p}\left(A_{n}\right)$ is defined by

$$
\kappa_{p}\left(A_{n}\right)=\left\|A_{n}\right\|_{p}\left\|A_{n}^{-1}\right\|_{p}
$$

where, by convention, $\left\|A_{n}^{-1}\right\|_{p}=\infty$ if $A_{n}$ is not invertible.

Let $a$ be a rational function without poles on the complex unit circle $\mathbb{T}=\{z \in$ $\mathbb{C}:|z|=1\}$. We denote by $\left\{a_{k}\right\}_{k \in \mathbb{Z}}$ the sequence of the Fourier coefficients of $a$, that

${ }^{*}$ Received by the editors on 14 October 1999. Accepted for publication on 29 November 1999. Handling Editor:Ludwig Elsner. This work was supported by DFG-Kooperationsprojekt 436 RUS 113/426 for German and Russian scientists within the "Memorandum of Understanding" between DFG and RFFI.

${ }^{\dagger}$ Faculty of Mathematics, Technische Universität Chemnitz, D - 09107 Chemnitz, Germany (aboettch@mathematik.tu-chemnitz.de).

${ }_{\ddagger}^{\ddagger}$ Faculty of Mechanics and Mathematics, Rostov-on-Don State University, Bolshaya Sadovaya 105, 344711 Rostov-on-Don, Russia (grudsky@aaanet.ru), partially supported by RFFI Grant 9801-01023. 
is,

$$
a_{n}=\frac{1}{2 \pi} \int_{0}^{2 \pi} a\left(e^{i \theta}\right) e^{-i n \theta} d \theta=\frac{1}{2 \pi i} \int_{\mathbb{T}} a(z) z^{-n-1} d z .
$$

The $n \times n$ Toeplitz matrix with the symbol $a$ is the matrix $T_{n}(a)=\left(a_{j-k}\right)_{j, k=0}^{n-1}$. If $\lambda \in \mathbb{C}$, then $T_{n}(a)-\lambda I=T_{n}(a-\lambda)$. It is well known that, for fixed $a$, the properties of $T_{n}(a-\lambda)$ depend very sensitively on $\lambda \in \mathbb{C}$. This paper is devoted to the behavior of the condition numbers $\kappa\left(T_{n}(a-\lambda)\right)$ as $n$ goes to infinity. Notice that $T_{n}(a)$ is a band matrix if and only if $a$ is of the form $a(z)=\sum_{k=-m}^{m} a_{k} z^{k}$.

Given an $n \times n$ matrix $A_{n}$, for instance, $A_{n}=T_{n}(a-\lambda)$, we let $\sigma_{1}\left(A_{n}\right) \leq \ldots \leq$ $\sigma_{n}\left(A_{n}\right)$ denote the singular values of $A_{n}$, that is, the eigenvalues of $\left(A_{n}^{*} A_{n}\right)^{1 / 2}$. The $l^{p}$ analogues of the singular values are the approximation numbers. For $j \in\{0,1, \ldots, n\}$, we denote by $\mathcal{F}_{j}^{(n)}$ the $n \times n$ matrices of rank at most $j$. Then the approximation numbers

$$
\sigma_{1}^{(p)}\left(A_{n}\right) \leq \ldots \leq \sigma_{n}^{(p)}\left(A_{n}\right)
$$

are defined by

$$
\sigma_{j}^{(p)}\left(A_{n}\right)=\operatorname{dist}_{p}\left(A_{n}, \mathcal{F}_{n-j}^{(n)}\right):=\inf \left\{\left\|A_{n}-F_{n}\right\|_{p}: F_{n} \in \mathcal{F}_{n-j}^{(n)}\right\} .
$$

It is well known that $\sigma_{j}^{(2)}\left(A_{n}\right)=\sigma_{j}\left(A_{n}\right)$. Moreover, we have

$$
\sigma_{1}^{(p)}\left(A_{n}\right)=\left\|A_{n}^{-1}\right\|_{p}^{-1}, \quad \sigma_{n}^{(p)}\left(A_{n}\right)=\left\|A_{n}\right\|_{p} .
$$

Consequently,

$$
\kappa_{p}\left(A_{n}\right)=\frac{\sigma_{n}^{(p)}\left(A_{n}\right)}{\sigma_{1}^{(p)}\left(A_{n}\right)}=\frac{\left\|A_{n}\right\|_{p}}{\sigma_{1}^{(p)}\left(A_{n}\right)} .
$$

In the case where $A_{n}=T_{n}(a-\lambda)$, the norms $\left\|A_{n}\right\|_{p}$ have a finite limit, the $p$ norm of the infinite Toeplitz matrix $T(a-\lambda)=\left(a_{j-k}-\lambda \delta_{j, k}\right)_{j, k=0}^{\infty}$. Thus, the asymptotic behavior of the condition numbers $\kappa\left(T_{n}(a-\lambda)\right)$ is entirely governed by the smallest approximation number $\sigma_{1}^{(p)}\left(T_{n}(a-\lambda)\right)$.

We orient the unit circle $\mathbb{T}$ counter-clockwise. Then the image $a(\mathbb{T})$ is a naturally oriented closed curve in the complex plane $\mathbb{C}$. For $\lambda \in \mathbb{C} \backslash a(\mathbb{T})$, we denote by wind $(a, \lambda)$ the winding number of the curve $a(\mathbb{T})$ about $\lambda$. Clearly, the integer wind $(a, \lambda)$ is constant on each connected component of $\mathbb{C} \backslash a(\mathbb{T})$.

A classical result by Gohberg and Feldman [12] says that

$$
\liminf _{n \rightarrow \infty} \sigma_{1}^{(p)}\left(T_{n}(a-\lambda)\right)>0
$$

or, equivalently,

$$
\limsup _{n \rightarrow \infty} \kappa_{p}\left(T_{n}(a-\lambda)\right)<\infty
$$


if and only if $\lambda \in \mathbb{C} \backslash a(\mathbb{T})$ and wind $(a, \lambda)=0$. Only recently, Roch and Silbermann [18] discovered that if $\lambda \in \mathbb{C} \backslash a(\mathbb{T})$ and wind $(a, \lambda)=k \neq 0$, then $|k|$ singular values of $T_{n}(a-\lambda)$ go to zero, while the remaining $n-|k|$ singular values stay away from zero (this is the so-called "splitting phenomenon"). In [4], this result was proved by completely different methods and extended to approximation numbers. Thus, for $\lambda \in \mathbb{C} \backslash a(\mathbb{T})$ and wind $(a, \lambda)=k$, we have

$$
\lim _{n \rightarrow \infty} \sigma_{|k|}^{(p)}\left(T_{n}(a-\lambda)\right)=0, \quad \liminf _{n \rightarrow \infty} \sigma_{|k|+1}^{(p)}\left(T_{n}(a-\lambda)\right)>0 .
$$

We here sharpen the last result as follows.

Theorem 1.1. If $\lambda \in \mathbb{C} \backslash a(\mathbb{T})$ and wind $(a, \lambda)=k \neq 0$, then there are constants $C \in(0, \infty)$ and $\alpha \in(0, \infty)$ independent of $n$ such that

$$
\sigma_{|k|}^{(p)}\left(T_{n}(a-\lambda)\right) \leq C e^{-\alpha n} \quad \text { for all } n \geq 1 .
$$

This theorem in conjunction with (2) implies that in the case wind $(a, \lambda) \neq 0$ the condition numbers increase at least exponentially:

Corollary 1.2. If $\lambda \in \mathbb{C} \backslash a(\mathbb{T})$ and wind $(a, \lambda) \neq 0$, then there exist constants $D \in(0, \infty)$ and $\alpha \in(0, \infty)$ independent of $n$ such that

$$
\kappa_{p}\left(T_{n}(a-\lambda)\right) \geq D e^{\alpha n} \quad \text { for all } n \geq 1 .
$$

It turns out that the constant $\alpha$ can be determined. Let $0<r_{\lambda}<1<R_{\lambda}<\infty$ be any two numbers such that $a(z)-\lambda$ has no zeros and no poles in the annulus $\left\{z \in \mathbb{C}: r_{\lambda} \leq|z| \leq R_{\lambda}\right\}$. We will show that Theorem 1.1 and Corollary 1.2 are true with

$$
\alpha=\min \left(\log \frac{1}{r_{\lambda}}, \log R_{\lambda}\right) .
$$

Of course, the constants $C$ and $D$ also depend on $\lambda$.

Corollary 1.2 provides us with a lower estimate for the condition numbers and raises the question about upper estimates. Let, for example, $a(z)=z$. Then $a(\mathbb{T})=\mathbb{T}$ and wind $(a, 0)=1$. Obviously, $T_{n}(a-0)=T_{n}(a)$ is not invertible and therefore $\kappa_{p}\left(T_{n}(a)\right)=\infty$ for all $n \geq 1$. This shows that nontrivial universal upper estimates do not exist.

The function $a-\lambda$ belongs to $L^{2}(\mathbb{T})$ and, by Parseval's equality,

$$
\left|a_{0}-\lambda\right|^{2}+\sum_{j \neq 0}\left|a_{j}\right|^{2}=\frac{1}{2 \pi} \int_{0}^{2 \pi}\left|a\left(e^{i \theta}\right)-\lambda\right|^{2} d \theta=\frac{1}{2 \pi}\|a-\lambda\|_{2}^{2} .
$$

The following is some kind of an upper estimate.

Theorem 1.3. If $\lambda \in \mathbb{C} \backslash a(\mathbb{T})$ and wind $(a, \lambda) \neq 0$, then

$$
\left\|T_{n}^{-1}(a-\lambda)\right\|_{p} \leq \frac{n}{\left|\operatorname{det} T_{n}(a-\lambda)\right|}\left(\frac{1}{\sqrt{2 \pi}}\|a-\lambda\|_{2}\right)^{n-1} .
$$


Since $n<e^{n / 10}$ for all sufficiently large $n$, we can put

$$
\beta=\frac{1}{10}+\log \left(\frac{1}{\sqrt{2 \pi}}\|a-\lambda\|_{2}\right)
$$

and, for $n$ large enough, rewrite (5) in the form

$$
\kappa_{p}\left(T_{n}(a-\lambda)\right) \leq \frac{1}{\left|\operatorname{det} T_{n}(a-\lambda)\right|} E e^{\beta n}
$$

with some constant $E \in(0, \infty)$ independent of $n$. Notice that formulas for the determinants of rationally generated Toeplitz matrices are available (see, e.g., [3], [8], $[9],[10])$.

The following example illustrates Corollary 1.2 and Theorem 1.3. Let $a(z)=$ $z-4 z^{-1}$ and $\lambda=0$. Since $a(z)=z^{-1}(z-2)(z+2)$, we see that wind $(a, 0)=-1$. Elementary computations give

$$
\operatorname{det} T_{n}(a)=\left\{\begin{array}{cccc}
2^{n} & \text { if } & n & \text { is even } \\
0 & \text { if } & n & \text { is odd }
\end{array}\right.
$$

From (4) we get $\|a\|_{2} / \sqrt{2 \pi}=\sqrt{17}$, and Theorem 1.3 so provides us with the estimate

$$
\left\|T_{n}^{-1}(a)\right\|_{p} \leq \frac{1}{\sqrt{17}} n\left(\frac{\sqrt{17}}{2}\right)^{n}<\frac{1}{4} n e^{0.724 n}<\frac{1}{4} e^{0.73 n}
$$

for all sufficiently large even $n$. On the other hand, Corollary 1.2 and (3) show that

$$
\left\|T_{n}^{-1}(a)\right\|_{p} \geq D e^{n(\log 2-0.001)}>D e^{0.69 n}
$$

with some constant $D>0$ for all $n$. In summary, there are $D_{1}, D_{2} \in(0, \infty)$ such that

$$
D_{1} e^{0.69 n} \leq \kappa_{p}\left(T_{n}(a)\right) \leq D_{2} e^{0.73 n} \text { for all even } n,
$$

whereas

$$
\kappa_{p}\left(T_{n}(a)\right)=\infty \text { for all odd } n .
$$

Let $\operatorname{sp} T_{n}(a)$ stand for the spectrum ( $=$ set of eigenvalues) of $T_{n}(a)$. The limiting set

$$
\Lambda(a)=\limsup _{n \rightarrow \infty} \operatorname{sp} T_{n}(a)
$$

is the set of all partial limits of the sequence $\left\{\operatorname{sp} T_{n}(a)\right\}_{n=1}^{\infty}$. In other words, $\lambda \in \Lambda(a)$ if and only if there are $n_{1}<n_{2}<n_{3}<\ldots$ and $\lambda_{n_{k}} \in \operatorname{sp} T_{n_{k}}(a)$ such that $\lambda_{n_{k}} \rightarrow \lambda$. The set $\Lambda(a)$ is known from the work of Schmidt and Spitzer [20], Hirschman [14], and Day [11]. It can be described in terms of only the function $a$, and one can show that $\Lambda(a)$ is either a single point or a finite union of analytic arcs which have at 
most their endpoints in common. Thus, $\Lambda(a)$ is always a "thin" set. If $a(z)=z$ and $a(z)=z-4 z^{-1}$ are as in the above examples, then $\Lambda(a)=\{0\}$ and $\Lambda(a)=[-4 i, 4 i]$, respectively.

Theorem 1.4. If $\lambda \in \mathbb{C} \backslash(a(\mathbb{T}) \cup \Lambda(a))$ and wind $(a, \lambda) \neq 0$, then there are constants $D_{1}, D_{2} \in(0, \infty)$ and $\alpha, \beta \in(0, \infty)$ independent of $n$ such that

$$
D_{1} e^{\alpha n} \leq \kappa_{p}\left(T_{n}(a-\lambda)\right) \leq D_{2} e^{\beta n}
$$

for all sufficiently large $n$.

As the following result shows, $\kappa_{2}\left(T_{n}(a-\lambda)\right)$ may grow arbitrarily fast on $\Lambda(a)$. We let $\mathbb{N}$ denote the natural numbers.

Theorem 1.5. Let $a(z)=z+z^{-1} / 4$. Then $a(\mathbb{T})$ is an ellipse with the foci -1 and 1 , and $\Lambda(a)=[-1,1]$. Given any function $\varphi: \mathbb{N} \rightarrow \mathbb{N}$, for example, $\varphi(n)=\exp \left(n^{n}\right)$, there exists a point $\lambda \in \Lambda(a)$ such that $\kappa_{p}\left(T_{n}(a-\lambda)\right)<\infty$ for all $n \geq 1$ but

$$
\kappa_{p}\left(T_{n_{k}}(a-\lambda)\right)>\varphi\left(n_{k}\right) \quad \text { for infinitely many } n_{k} .
$$

The results quoted above provide an idea of the behavior of $\kappa_{p}\left(T_{n}(a-\lambda)\right)$ for $\lambda$ in the connected components of $\mathbb{C} \backslash a(\mathbb{T})$ whose winding number is nonzero. As already said, Gohberg and Feldman showed that $\kappa_{p}\left(T_{n}(a-\lambda)\right)$ remains bounded on the other connected components of $\mathbb{C} \backslash a(\mathbb{T})$. In [6], we proved that if wind $(a, \lambda)=0$, then the condition numbers $\kappa_{p}\left(T_{n}(a-\lambda)\right)$ even converge to a finite limit as $n \rightarrow \infty$.

The behavior of $\kappa_{p}\left(T_{n}(a-\lambda)\right)$ for $\lambda \in a(\mathbb{T})$ is more intricate. Much work on this problem has been done in the case where $a$ is real-valued. Let us assume that $a$ is a real-valued nonconstant continuous function on $\mathbb{T}$. Then the matrices $T_{n}(a)$ are Hermitian and $\Lambda(a)$ can be shown to be the line segment $a(\mathbb{T})=[\min a, \max a]=$ : $[m, M]$. If $\lambda$ does not belong to this segment, then it is easy to prove that $\kappa_{2}\left(T_{n}(a-\lambda)\right)$ remains bounded as $n \rightarrow \infty$ (Brown-Halmos theorem). If $\lambda$ is one of the endpoints $m$ or $M$ of the segment $[m, M]$, then $a(z)-\lambda$ has zeros on $\mathbb{T}$ and all these zeros are of even order. Suppose all these zeros have finite orders and let $2 \alpha$ be the maximal order. The outgrowth of the works [13], [16], [17], [21], [23] is that there are constants $D_{1}, D_{2} \in(0, \infty)$ such that

$$
D_{1} n^{2 \alpha} \leq \kappa_{2}\left(T_{n}(a-\lambda)\right) \leq D_{2} n^{2 \alpha} .
$$

Rosenblatt [19] was probably the first to understand that if $\lambda \in\{m, M\}$ and $a(z)-\lambda$ vanishes identically on some subarc of $\mathbb{T}$, then $\kappa_{2}\left(T_{n}(a-\lambda)\right)$ increases exponentially. Serra [22] observed that if $\lambda \in\{m, M\}$ then always $\kappa_{2}\left(T_{n}(a-\lambda)\right)=O\left(e^{\alpha n}\right)$ for some constant $\alpha \in(0, \infty)$ as $n \rightarrow \infty$.

Some general results on the condition numbers of non-Hermitian Toeplitz matrices are in [5]. There we proved, in particular, that if $a$ is rational and $\lambda \in a(\mathbb{T})$, then necessarily $\kappa_{2}\left(T_{n}(a-\lambda)\right) \geq D n$ with some constant $D \in(0, \infty)$ independent of $n$. Moreover, if $a(z)-\lambda$ has a zero of order $\alpha \in \mathbb{N}$, then even $\kappa_{2}\left(T_{n}(a-\lambda)\right) \geq D n^{\alpha}$ with some constant $D \in[0, \infty)$ which does not depend on $n$. 
Finally, suppose $a$ is both rational and real-valued. Then again $\Lambda(a)=a(\mathbb{T})=$ $[m, M]$. Rational functions cannot vanish on an open arc unless they vanish identically. Thus, we cannot expect that $\kappa_{2}\left(T_{n}(a-\lambda)\right)$ grows exponentially for $\lambda \in\{m, M\}$. From (6) we infer that indeed $\kappa_{2}\left(T_{n}(a-\lambda)\right)$ increases at most polynomially if $\lambda$ is one of the endpoints $m$ and $M$. As the following result shows, things change dramatically for points $\lambda$ in the interior of $[m, M]$.

Theorem 1.6. Let $a(z)=z+z^{-1}$. Then $a(\mathbb{T})=\Lambda(a)=[-2,2]$, and given any function $\varphi: \mathbb{N} \rightarrow \mathbb{N}$, there exist an $\lambda \in(-2,2)$ such that

$$
\kappa_{p}\left(T_{n}(a-\lambda)\right)<\infty \text { for all } n \geq 1
$$

but

$$
\kappa_{p}\left(T_{n_{k}}(a-\lambda)\right)>\varphi\left(n_{k}\right) \quad \text { for infinitely many } n_{k} .
$$

We will establish several new results on the condition numbers $\kappa_{2}\left(T_{n}(a-\lambda)\right)$ for $\lambda \in a(\mathbb{T})$.

Suppose that $a(t)-\lambda$ has only one zero on $\mathbb{T}$. We label each connected component of $\mathbb{C} \backslash a(\mathbb{T})$ with the winding number of $a(\mathbb{T})$ about the points of the component. Our assumption that $a(t)-\lambda$ has only a single zero amounts to the requirement that $\lambda$ is a point on $a(\mathbb{T})$ and that $a(\mathbb{T})$ passes through $\lambda$ exactly once. In particular, $a(\mathbb{T})$ has no self-intersection at $\lambda$. In a neighborhood of $\lambda$, the curve $a(\mathbb{T})$ is an oriented analytic arc $\gamma_{\lambda}$. Let $M_{\lambda}^{+}$and $M_{\lambda}^{-}$be the connected components of $\mathbb{C} \backslash a(\mathbb{T})$ which lie on the left and the right of $\gamma_{\lambda}$, respectively.

In what follows we write $x_{n} \sim y_{n}$ if $y_{n} \neq 0$ for all sufficiently large $n$ and $\lim \left(x_{n} / y_{n}\right)=1$, while $x_{n} \simeq y_{n}$ means that $y_{n} \neq 0$ and $1 / C \leq x_{n} / y_{n} \leq C$ with some constant $C \in(0, \infty)$ for all $n$ large enough.

THEOREM 1.7. Let a be a rational function without poles on $\mathbb{T}$ and let $\lambda \in a(\mathbb{T})$. Suppose $b(t):=a(t)-\lambda$ has exactly one zero $t_{0} \in \mathbb{T}$ and let $\beta$ be the order of this zero. Furthermore, let $m$ denote the winding number of $M_{\lambda}^{+}$. Then

$$
\kappa_{2}\left(T_{n}(a-\lambda)\right) \simeq n^{\beta} \quad \text { if } \quad-\left[\frac{\beta}{2}\right] \leq m \leq\left[\frac{\beta+1}{2}\right],
$$

and there are constants $C>0$ and $\alpha>0$ such that

$$
\kappa_{2}\left(T_{n}(a-\lambda)\right) \geq C e^{\alpha n} \quad \text { if } \quad m<-\left[\frac{\beta}{2}\right] \quad \text { or } \quad m>\left[\frac{\beta+1}{2}\right] .
$$

We denote by $S(a)$ the points of $a(\mathbb{T})$ at which $a(\mathbb{T})$ has self-intersections. Equivalently, $\lambda \in S(a)$ if and only if $a(t)-\lambda$ has at least two different zeros on $\mathbb{T}$. In many cases $S(a)$ is a finite subset of $a(\mathbb{T})$, but as functions of the form $a(t)=d(B(t))$ with a rational function $d$ and a finite Blaschke product $B$ show, $S(a)$ may also coincide with all of $a(\mathbb{T})$. If $\lambda \in a(\mathbb{T}) \backslash S(a)$, then $a(t)-\lambda$ has a single zero on $\mathbb{T}$. The order of this zero will be denoted by $\beta(\lambda)$. 
The spectrum of the Toeplitz operator $T(a)$ given on $l^{2}$ by the infinite Toeplitz matrix $\left(a_{j-k}\right)_{j, k=0}^{\infty}$ is

$$
\operatorname{sp} T(a)=a(\mathbb{T}) \cup\{\mu \in \mathbb{C} \backslash a(\mathbb{T}): \text { wind }(a, \mu) \neq 0\} .
$$

Let $\partial \operatorname{sp} T(a)$ stand for the boundary of $\operatorname{sp} T(a)$.

Theorem 1.8. If $\lambda \in a(\mathbb{T}) \backslash S(a)$ is located on $\partial \operatorname{sp} T(a)$, then

$$
\kappa_{2}\left(T_{n}(a-\lambda) \simeq n^{\beta(\lambda)} .\right.
$$

Theorem 1.9. Suppose $\beta(\lambda)=1$ for all $\lambda \in a(\mathbb{T}) \backslash S(a)$. Then

$$
\kappa_{2}\left(T_{n}(a-\lambda)\right) \simeq n
$$

if $\lambda \in a(\mathbb{T}) \backslash S(a)$ lies on $\partial \operatorname{sp} T(a)$, whereas

$$
\kappa_{2}\left(T_{n}(a-\lambda)\right) \text { inceases at least exponentially }
$$

if $\lambda \in a(\mathbb{T}) \backslash S(a)$ is not located on $\partial \operatorname{sp} T(a)$.

The rest of the paper contains the proofs of the theorems stated above. The two main ingredients to the proof of Theorem 1.1 are the so-called finite section method for Toeplitz operators on certain spaces with exponential weights on the one hand and the approach to the approximation numbers developed in [4] on the other. Corollary 1.2 is an immediate consequence of Theorem 1.1. Theorem 1.3 is nothing but a simple application of Hadamard's inequality. The proof of Theorem 1.4 is based on Theorem 1.3 and on Day's formulas for the determinants of $T_{n}(a-\lambda)$ and the limiting set $\Lambda(a)$. To prove Theorems 1.5 and 1.6, we first make use of the fact that the eigenvalues of tridiagonal Toeplitz matrices are explicitly available, and we then find the desired $\lambda$ by employing a standard construction of number theory. Theorem 1.7 will be derived from some rather subtle results of [8]. Theorems 1.8 and 1.9 are simple consequences of Theorem 1.7. In the last section of the paper we present a few additional examples and results on the condition numbers of Toeplitz matrices whose symbols have zeros.

The paper has an appendix. This appendix does not contain terribly new results, but it tells a nice story we have not yet seen in this form in the literature.

2. Spaces with exponential weight. In this section we prepare the proof of Theorem 1.1. The results of this section are known to specialists. As we do not know an explicit reference, we outline the proofs for the reader's convenience.

Given a real number $\beta$, we denote by $l^{p, \beta}$ the Banach space of all sequences $x=\left\{x_{n}\right\}_{n=0}^{\infty}$ for which

$$
\begin{aligned}
& \|x\|_{p, \beta}^{p}=\sum_{n=0}^{\infty} e^{p n \beta}\left|x_{n}\right|^{p}<\infty \quad(1 \leq p<\infty), \\
& \|x\|_{\infty, \beta}=\sup _{n \geq 0} e^{n \beta}\left|x_{n}\right|<\infty \quad(p=\infty) .
\end{aligned}
$$


For $n \in \mathbb{N}$, let $P_{n}$ and $Q_{n}$ be the projections

$$
\begin{aligned}
& P_{n}:\left\{x_{0}, x_{1}, x_{2}, \ldots\right\} \mapsto\left\{x_{0}, x_{1}, \ldots, x_{n-1}, 0,0, \ldots\right\}, \\
& Q_{n}:\left\{x_{0}, x_{1}, x_{2}, \ldots\right\} \mapsto\left\{0,0, \ldots, 0, x_{n}, x_{n+1}, \ldots\right\} .
\end{aligned}
$$

The image of $P_{n}: l^{p, \beta} \rightarrow l^{p, \beta}$ will be denoted by $l_{n}^{p, \beta}$. We identify $l_{n}^{p, \beta}$ and the space $\mathbb{C}^{n}$ with the $l^{p, \beta}$ norm. Given Banach spaces $X$ and $Y$, we let $\mathcal{B}(X, Y)$ stand for the Banach space of all bounded linear operators of $X$ to $Y$, and we abbreviate $\mathcal{B}(X, X)$ to $\mathcal{B}(X)$.

For a rational function $a$ without poles on $\mathbb{T}$, we denote by $T(a)$ and $H(a)$ the infinite Toeplitz and Hankel matrices generated by $a$ :

$$
T(a)=\left(a_{j-k}\right)_{j, k=0}^{\infty}, \quad H(a)=\left(a_{j+k+1}\right)_{j, k=0}^{\infty} .
$$

Let $r \in(0,1)$ and $R \in(1, \infty)$ be any numbers such that $a(z)$ has neither zeros nor poles in the annulus $\{z \in \mathbb{C}: r \leq|z| \leq R\}$. Clearly, there is an $\varepsilon>0$ such that $a(z)$ is analytic and nonzero in the larger annulus $\{z \in \mathbb{C}: r-\varepsilon \leq|z| \leq R+\varepsilon\}$. From (1) we therefore see that if $n \geq 0$, then

$$
\left|a_{n}\right| \leq M_{1} \frac{1}{(R+\varepsilon)^{n}}, \quad\left|a_{-n}\right| \leq M_{2}(r-\varepsilon)^{n} .
$$

where $M_{1}$ and $M_{2}$ is the $L^{\infty}$ norm of $a$ on the circle of the radius $R+\varepsilon$ and $r-\varepsilon$, respectively. Put

$$
\alpha=\min \left(\log \frac{1}{r}, \log R\right) .
$$

Proposition 2.1. If $|\beta| \leq \alpha$ and $1 \leq p \leq \infty$, then the matrices $T(a)$ and $H(a)$ induce bounded operators on $l^{p, \beta}$.

Proof. Let $\chi_{n}(z)=z^{n}$. We then have $T(a)=\sum_{n \in \mathbb{Z}} a_{n} T\left(\chi_{n}\right)$. From (9) and (10) we infer that $\sum_{n \in \mathbb{Z}}\left|a_{n}\right| e^{|n||\beta|}<\infty$, and it can be verified straightforwardly that $\left\|T\left(\chi_{n}\right)\right\|_{p, \beta}=O\left(e^{|n||\beta|}\right)$. This shows that $T(a) \in \mathcal{B}\left(l^{p, \beta}\right)$. The proof for $H(a)$ is analogous.

Proposition 2.2. Let $|\beta| \leq \alpha$ and $1 \leq p \leq \infty$. If wind $(a, 0)=0$, then $T(a)$ is invertible on $l^{p, \beta}$.

Proof. On writing $a$ as the quotient of two polnomials, it is easily seen that $a$ can be represented in the form

$$
a(t)=a_{-}(t) a_{+}(t) \quad(t \in \mathbb{T})
$$

where $a_{-}(z)$ has no zeros and poles in $\{z \in \mathbb{C}:|z| \geq 1\}$ and $a_{+}(z)$ has no zeros and poles in $\{z \in \mathbb{C}:|z| \leq 1\}$. The representation (11) is called a Wiener-Hopf factorization. The Fourier coefficients of $a_{-}, a_{-}^{-1}, a_{+}, a_{+}^{-1}$ admit estimates analogous to (9), and therefore we obtain as in the proof of Proposition 2.1 that $T\left(a_{-}\right), T\left(a_{-}^{-1}\right)$, $T\left(a_{+}\right), T\left(a_{+}^{-1}\right)$ are bounded on $l^{p, \beta}$. One can now show that $T(a)=T\left(a_{-}\right) T\left(a_{+}\right)$and that $T\left(a_{+}^{-1}\right) T\left(a_{-}^{-1}\right)$ is the inverse operator of $T(a)$. 
Lemma 2.3. If $\beta>0$ and $1 \leq p \leq \infty$, then

$$
\begin{aligned}
\left\|P_{n}\right\|_{\mathcal{B}\left(l^{p, 0}, l^{p, \beta}\right)} \leq e^{n \beta}, & \left\|P_{n}\right\|_{\mathcal{B}\left(l^{p,-\beta}, l^{p, 0}\right)} \leq e^{n \beta} \\
\left\|Q_{n}\right\|_{\mathcal{B}\left(l^{p, \beta}, l^{p, 0}\right)} \leq e^{-n \beta}, & \left\|Q_{n}\right\|_{\mathcal{B}\left(l^{p, 0}, l^{p,-\beta}\right)} \leq e^{-n \beta} .
\end{aligned}
$$

Proof. Straightforward. $\square$

Theorem 2.4. Let $|\beta| \leq \alpha$ and $1 \leq p \leq \infty$. If wind $(a, 0)=0$, then

$$
\limsup _{n \rightarrow \infty}\left\|T_{n}^{-1}(a)\right\|_{\mathcal{B}\left(l_{n}^{p, \beta}\right)}<\infty .
$$

Proof. The dual space of $l_{n}^{p, \beta}$ is $l_{n}^{q,-\beta}(1 / p+1 / q=1)$. It therefore suffices to prove the theorem for $\beta \geq 0$. In the case $\beta=0$, the theorem is Gohberg and Feldman's [12]. The $\beta=0$ result implies in particular that $T_{n}(a)$ is invertible for all sufficiently large $n$. Now let $x \in l_{n}^{p, \beta}$. We then have

$$
\begin{aligned}
& \left\|T_{n}^{-1}(a) x\right\|_{p, \beta} \\
& \quad \leq\left\|T_{n}^{-1}(a) x-P_{n} T^{-1}(a) P_{n} x\right\|_{p, \beta}+\left\|P_{n}\right\|_{\mathcal{B}\left(l^{p, \beta}\right)}\left\|T^{-1}(a)\right\|_{\mathcal{B}\left(l^{p, \beta}\right)}\|x\|_{p, \beta} .
\end{aligned}
$$

By Proposition 2.2, the second term on the right of (15) does not exceed $M_{2}\|x\|_{p, \beta}$ with some constant $M_{2}<\infty$ independent of $n$. Taking into account (12) we obtain the estimate

$$
\left\|T_{n}^{-1}(a) x-P_{n} T^{-1}(a) P_{n} x\right\|_{p, \beta} \leq e^{n \beta}\left\|T_{n}^{-1}(a) x-P_{n} T^{-1}(a) P_{n} x\right\|_{p, 0}
$$

for the first term on the right of (15), and since

$$
\left\|T_{n}^{-1}(a)\right\|_{\mathcal{B}\left(l_{n}^{p, 0}\right)} \leq C<\infty
$$

for all sufficiently large $n$, it follows that (16) is not larger than

$$
\begin{aligned}
& C e^{n \beta}\left\|P_{n} x-P_{n} T(a) P_{n} T^{-1}(a) P_{n} x\right\|_{p, 0} \\
& \quad=C e^{n \beta}\left\|P_{n} T(a) Q_{n} T^{-1}(a) P_{n} x\right\|_{p, 0} \\
& \quad \leq C e^{n \beta}\|T(a)\|_{\mathcal{B}\left(l^{p, 0}\right)}\left\|Q_{n}\right\|_{\mathcal{B}\left(l^{p, \beta}, l^{p, 0}\right)}\left\|T^{-1}(a)\right\|_{\mathcal{B}\left(l^{p, \beta}\right)}\|x\|_{p, \beta}
\end{aligned}
$$

Propositions 2.1 and 2.2 in conjunction with (13) imply that (17) can be estimated from above by $M_{1}\|x\|_{p, \beta}$ with some $M_{1}<\infty$ independent of $n$. In summary,

$$
\left\|T_{n}^{-1}(a) x\right\|_{p, \beta} \leq\left(M_{1}+M_{2}\right)\|x\|_{p, \beta} \quad \text { for all } \quad x \in l_{n}^{p, \beta},
$$

which completes the proof. $\square$

3. Exponential decay of approximation numbers. The purpose of this section is to prove Theorem 1.1.

Thus, let $\lambda \in \mathbb{C} \backslash a(\mathbb{T})$ and suppose wind $(a, \lambda)=k \neq 0$. For the sake of definiteness, let us assume that $k>0$; the case $k<0$ can be reduced to this case by passage 
to adjoints. Put $b=a-\lambda$, choose $r=r_{\lambda} \in(0,1)$ and $R=R_{\lambda} \in(1, \infty)$ so that $b(z)$ has no poles and zeros in $\{z \in \mathbb{C}: r \leq|z| \leq R\}$, and define $\alpha=\alpha_{\lambda}$ by (10).

We can write $b=\chi_{k} c$ where $\chi_{k}(z)=z^{k}$ and wind $(c, 0)=0$. Define $\widetilde{c}$ by $\widetilde{c}(t)=$ $c(1 / t)$ for $t \in \mathbb{T}$. The Hankel operator $H(\widetilde{c})$ is given by the matrix $\left(c_{-j-k-1}\right)_{j, k=0}^{\infty}$. We have

$$
T_{n}(b)=T_{n}\left(\chi_{k}\right) T_{n}(c)+P_{n} H\left(\chi_{k}\right) H(\widetilde{c}) P_{n} ;
$$

this can be verified directly by computing the $j k$ entries of each side or can be derived from a more general result on the multiplication of finite Toeplitz matrices (see, e.g., [9, Proposition 2.12]). Hence,

$$
\sigma_{k}^{(p)}\left(T_{n}(b)\right)=\operatorname{dist}_{p}\left(T_{n}\left(\chi_{k}\right) T_{n}(c)+P_{n} H\left(\chi_{k}\right) H(\widetilde{c}) P_{n}, \mathcal{F}_{n-k}^{(n)}\right) .
$$

If $n$ is large enough, then $T_{n}(c)$ is invertible and

$$
\left\|T_{n}^{-1}(c)\right\|_{\mathcal{B}\left(l_{n}^{p,-\alpha}\right)} \leq M<\infty
$$

due to Theorem 2.4. Since $\operatorname{dist}_{p}\left(A B, \mathcal{F}_{n-k}^{(n)}\right) \leq\|B\|_{p} \operatorname{dist}_{p}\left(A, \mathcal{F}_{n-k}^{(n)}\right)$, we obtain

$$
\sigma_{k}^{(p)}\left(T_{n}(b)\right) \leq\left\|T_{n}(c)\right\|_{p} \operatorname{dist}_{p}\left(A_{n}, \mathcal{F}_{n-k}^{(n)}\right)
$$

with

$$
A_{n}=T_{n}\left(\chi_{k}\right)+P_{n} H\left(\chi_{k}\right) H(\widetilde{c}) P_{n} T_{n}^{-1}(c) .
$$

Obviously, $\left\|T_{n}(c)\right\|_{p} \leq\|c\|_{W}:=\sum_{j \in \mathbb{Z}}\left|c_{j}\right|$. Because $A_{n}=A_{n} Q_{n-k}+A_{n} P_{n-k}$ and $A_{n} P_{n-k}$ is in $\mathcal{F}_{n-k}^{(n)}$, we get from (19) that

$$
\sigma_{k}^{(p)}\left(T_{n}(b)\right) \leq\|c\|_{W} \operatorname{dist}_{p}\left(A_{n} Q_{n-k}, \mathcal{F}_{n-k}^{(n)}\right) \leq\|c\|_{W}\left\|A_{n} Q_{n-k}\right\|_{p} .
$$

As $T_{n}\left(\chi_{k}\right) Q_{n-k}=0$, it follows that

$$
\begin{aligned}
\left\|A_{n} Q_{n-k}\right\|_{p} & =\left\|P_{n} H\left(\chi_{k}\right) H(\widetilde{c}) P_{n} T_{n}^{-1}(c) Q_{n-k}\right\|_{p} \\
& \leq\left\|H\left(\chi_{k}\right) H(\widetilde{c})\right\|_{\mathcal{B}\left(l^{p,-\alpha}, l^{p}\right)}\left\|T_{n}^{-1}(c)\right\|_{\mathcal{B}\left(l_{n}^{p,-\alpha}\right)}\left\|Q_{n-k}\right\|_{\mathcal{B}\left(l^{p}, l^{p,-\alpha}\right)} .
\end{aligned}
$$

By Proposition 2.1, $H(\widetilde{c})$ maps $l^{p,-\alpha}$ boundedly into itself. Since $H\left(\chi_{k}\right)$ has only finitely many nonzero entries, we arrive at the conclusion that the first factor of (21) is finite. The middle factor of (21) remains bounded by virtue of (18). From (13) we finally see that the third factor of $(21)$ is $O\left(e^{-n \alpha}\right)$. Thus, by (20),

$$
\sigma_{k}^{(p)}\left(T_{n}(b)\right)=O\left(e^{-n \alpha}\right) \quad \text { as } \quad n \rightarrow \infty,
$$

which completes the proof of Theorem 1.1. 
4. Upper estimates. This section contains the proof of Theorem 1.3. Let $b=a-\lambda, r, R, \alpha$ be as in Section 3. We abbreviate $\operatorname{det} T_{n}(b)$ to $D_{n}(b)$. What we must show is that

$$
\left\|T_{n}^{-1}(b)\right\|_{p} \leq \frac{n}{\left|D_{n}(b)\right|}\left(\frac{1}{\sqrt{2 \pi}}\|b\|_{2}\right)^{n-1}
$$

There is nothing to prove in case $D_{n}(b)=0$. So assume $D_{n}(b) \neq 0$. Then

$$
T_{n}^{-1}(b)=\frac{1}{D_{n}(b)} A_{n}(b),
$$

where $A_{n}(b)=\left(\Delta_{j k}^{(n)}(b)\right)_{j, k=1}^{n}$ and $\Delta_{j k}^{(n)}(b)$ is $(-1)^{j+k}$ times the determinant of the matrix arising from $T_{n}(b)$ by deleting the $k$ th row and the $j$ th column. By Hadamard's inequality (see, e.g., [2, p. 3]) and by (4),

$$
\left|\Delta_{j k}^{(n)}(a)\right| \leq\left[\left(\sum_{l \in \mathbb{Z}}\left|b_{l}\right|^{2}\right)^{1 / 2}\right]^{n-1}=\left(\frac{1}{\sqrt{2 \pi}}\|b\|_{2}\right)^{n-1}
$$

Estimating $\left\|A_{n}(b)\right\|_{p}$ by the $l^{p}$ analogue of the Frobenius norm we obtain, with obvious modifications for $p=1$ and $p=\infty$,

$$
\left\|A_{n}(b)\right\|_{p} \leq\left(\sum_{j=1}^{n}\left(\sum_{k=1}^{n}\left|\Delta_{j k}^{(n)}\right|^{q}\right)^{p / q}\right)^{1 / p}=n\left(\frac{1}{\sqrt{2 \pi}}\|b\|_{2}\right)^{n-1}
$$

This completes the proof of Theorem 1.3.

5. Outside the limiting set. The proof of Theorem 1.4 is based on two results by Day [10], [11].

Suppose first that $T_{n}(a)$ is a triangular matrix. Then $\Lambda(a)=\left\{a_{0}\right\}$. So let $\lambda \neq a_{0}$. It follows that $D_{n}(b)=b_{0}^{n}=\left(a_{0}-\lambda\right)^{n} \neq 0$, and (22) yields

$$
\left\|T_{n}^{-1}(b)\right\|_{p} \leq \frac{n}{\left|b_{0}\right|^{n}}\left(\frac{1}{\sqrt{2 \pi}}\|b\|_{2}\right)^{n-1} \leq D_{2} e^{\beta n}
$$

with certain $D_{2} \in(0, \infty)$ and $\beta \in(0, \infty)$ independent of $n$.

So assume $T_{n}(a)$ is not triangular. Then $a(z)$ can be written in the form

$$
a(z)=\gamma \prod_{j=1}^{s}\left(z-\varrho_{j}\right) \prod_{j=1}^{g}\left(1-\frac{z}{\mu_{j}}\right)^{-1} \prod_{j=1}^{h}\left(z-\nu_{j}\right)^{-1}
$$

where $\gamma \neq 0, s \geq 1, g \geq 1, h \geq 1$ and

$$
\left|\mu_{j}\right|>1 \quad(j=1, \ldots, g), \quad\left|\nu_{j}\right|<1 \quad(j=1, \ldots, h) .
$$

We can without loss of generality assume that $\gamma=1$. 
THEOREM 5.1. (DAY [10]) Let a be as above and suppose the zeros $\varrho_{1}, \ldots, \varrho_{s}$ are pairwise distinct. If $s<h$, then $D_{n}(a)=0$. If $s \geq h$, then

$$
D_{n}(a)=\sum_{M} C_{M} \omega_{M}^{n}
$$

where the sum is over all $\left(\begin{array}{l}s \\ h\end{array}\right)$ subsets $M \subset\{1, \ldots, s\}$ of cardinality $|M|=h$ and, with

$$
\bar{M}=\{1, \ldots, s\} \backslash M, \quad G=\{1, \ldots, g\}, \quad H=\{1, \ldots, h\},
$$

the constants are given by

$$
\begin{aligned}
\omega_{M} & =(-1)^{s-h} \prod_{j \in \bar{M}} \varrho_{j} \\
C_{M} & =\prod_{\substack{j \in \bar{M} \\
l \in H}}\left(\varrho_{j}-\nu_{l}\right) \prod_{\substack{i \in G \\
k \in M}}\left(\mu_{i}-\varrho_{k}\right) \prod_{\substack{i \in G \\
l \in H}}\left(\mu_{i}-\nu_{l}\right)^{-1} \prod_{\substack{j \in \bar{M} \\
k \in M}}\left(\varrho_{j}-\varrho_{k}\right)^{-1} .
\end{aligned}
$$

In order to determine the limiting set $\Lambda(a)$, we have to consider

$$
a(z)-\lambda=F_{m}(z, \lambda) \prod_{j=1}^{g}\left(1-\frac{z}{\mu_{j}}\right)^{-1} \prod_{j=1}^{h}\left(z-\nu_{j}\right)^{-1}
$$

with

$$
F_{m}(z, \lambda):=\prod_{j=1}^{s}\left(z-\varrho_{j}\right)-\lambda \prod_{j=1}^{g}\left(1-\frac{z}{\mu_{j}}\right) \prod_{j=1}^{h}\left(z-\nu_{j}\right)
$$

If $\lambda \neq 0$ and $\lambda \neq \prod_{j=1}^{g}\left(-\mu_{j}\right)$, then the polynomial $F_{m}(\cdot, \lambda)$ has the degree $m:=$ $\max (s, g+h)$.

TheOREM 5.2. (DAY [11]) Let a be the function introduced above. For $\lambda \neq 0$ and $\lambda \neq \prod_{j=1}^{g}\left(-\mu_{j}\right)$, label the zeros $z_{1}(\lambda), \ldots, z_{m}(\lambda)$ of $F_{m}(z, \lambda)$ so that

$$
\left|z_{1}(\lambda)\right| \leq\left|z_{2}(\lambda)\right| \leq \ldots \leq\left|z_{m}(\lambda)\right|
$$

Then

$$
\Lambda(a)=\operatorname{clos}\left\{\lambda \in \mathbb{C} \backslash\left\{0, \prod_{j=1}^{g}\left(-\mu_{j}\right)\right\}:\left|z_{g}(\lambda)\right|=\left|z_{g+1}(\lambda)\right|\right\}
$$

We now proceed to the proof of Theorem 1.4. Let $E(a)$ denote the set of all $\lambda \in \mathbb{C}$ for which $F_{m}(\cdot, \lambda)$ has multiple zeros. If $\lambda \in E(a)$, then $a(z)=\lambda$ and $a^{\prime}(z)=0$ for some $z \in \mathbb{C}$. As $a^{\prime}$ has only finitely many zeros in $\mathbb{C}$, it follows that $E(a)$ must be a finite set.

Suppose $\lambda \in \mathbb{C} \backslash(a(\mathbb{T}) \cup \Lambda(a) \cup E(a))$ and wind $(a, \lambda) \neq 0$. Asssume also that $\lambda \neq 0$ and $\lambda \neq \prod_{j=1}^{g}\left(-\mu_{j}\right)$. Then, by Theorem 5.2, $\left|z_{g}(\lambda)\right|<\left|z_{g+1}(\lambda)\right|$. Since 
the zeros $z_{1}(\lambda), \ldots, z_{m}(\lambda)$ of $a-\lambda$ are paiwise distinct for $\lambda \notin E(a)$, we can have recourse to Theorem 5.1 to compute $D_{n}(a-\lambda)$. The role of the numbers $\varrho_{1}, \ldots, \varrho_{s}$ of Theorem 5.1 is now played by the zeros $z_{1}(\lambda), \ldots, z_{m}(\lambda)$. Put $M_{0}=\{1,2, \ldots, h\}$. Since $\left|z_{g}(\lambda)\right|<\left|z_{g+1}(\lambda)\right|$, it follows that

$$
\left|\omega_{M_{0}}\right|=\left|z_{g+1}(\lambda)\right| \ldots\left|z_{m}(\lambda)\right|
$$

is strictly larger than all other $\left|\omega_{M}\right|$ 's. Consequently,

$$
\left|D_{n}(a-\lambda)\right|=\left|C_{M_{0}}\right|\left|\omega_{M_{0}}\right|^{n}\left(1+O\left(\delta_{\lambda}^{n}\right)\right)
$$

with some constant $\delta_{\lambda} \in(0,1)$. This implies that

$$
\frac{1}{\left|D_{n}(a-\lambda)\right|} \leq D(\lambda) e^{\beta(\lambda) n}
$$

with certain constants $D(\lambda) \in(0, \infty)$ and $\beta(\lambda) \in(0, \infty)$ independent of $n$. Combining (23) and Theorem 1.3 we arrive at the upper estimate of Theorem 1.4.

We are left with the case where $\lambda_{0} \in \mathbb{C} \backslash(a(\mathbb{T}) \cup \Lambda(a))$, wind $\left(a, \lambda_{0}\right) \neq 0$, but

$$
\lambda_{0} \in E(a) \cup\left\{0, \prod_{j=1}^{g}\left(-\mu_{j}\right)\right\} .
$$

Since $E(a)$ is finite, there is a small circle,$=\left\{\lambda \in \mathbb{C}:\left|\lambda-\lambda_{0}\right|=\varepsilon\right\}$ such that

$$
\lambda \notin a(\mathbb{T}) \cup \Lambda(a) \cup E(a) \cup\left\{0, \prod_{j=1}^{g}\left(-\mu_{j}\right)\right\}
$$

for all $\lambda \in$,. For the points $\lambda$ on the circle, we have the estimate (23). The zeros $z_{1}(\lambda), \ldots, z_{m}(\lambda)$ are continuous functions of $\lambda \in$, . From the explicit form of the constants in Theorem 5.1 we therefore see that the constants $D(\lambda)$ and $\beta(\lambda)$ in $(23)$ can also be chosen to be continuous functions of $\lambda$. Thus, since, is compact, there are constants $D \in(0, \infty)$ and $\beta \in(0, \infty)$ such that

$$
\frac{1}{\left|D_{n}(a-\lambda)\right|} \leq D e^{\beta n}
$$

for all $\lambda \in$,. If $n$ is sufficiently large, then, by the definition of $\Lambda(a), D_{n}(a-\lambda) \neq 0$ for all $\lambda \in \mathbb{C}$ satisfying $\left|\lambda-\lambda_{0}\right| \leq \varepsilon$. Hence $1 / D_{n}(a-\lambda)$ is an analytic function of $\lambda$ on the disk $\Delta:=\left\{\lambda \in \mathbb{C}:\left|\lambda-\lambda_{0}\right| \leq \varepsilon\right\}$. From the maximum modulus principle we therefore deduce that (24) holds for all $\lambda \in \Delta$ and, in particular, for $\lambda=\lambda_{0}$. This in conjunction with Theorem 1.3 gives the upper estimate in Theorem 1.4.

As the lower estimate of Theorem 1.4 follows from Corollary 1.2, the proof of Theorem 1.4 is complete. 
6. Inside the limiting set. This section is devoted to the proofs of Theorems 1.5 and 1.6.

Thus, let first $a(z)=z+z^{-1} / 4$. Because

$$
a\left(e^{i \theta}\right)=(1+1 / 4) \cos \theta+i(1-1 / 4) \sin \theta,
$$

it is clear that $a(\mathbb{T})$ is an ellipse with the foci -1 and 1 . The set $\Lambda(a)$ can be determined using Theorem 5.2, but since $T_{n}(a)$ is tridiagonal, we can also find $\Lambda(a)$ from computing the eigenvalues of $T_{n}(a)$. Namely, it is well known (see, e.g., [13]) that if

$$
a(z)=a_{-1} z^{-1}+a_{0}+a_{1} z,
$$

then the eigenvalues of $T_{n}(a)$ are

$$
\lambda_{j}\left(T_{n}(a)\right)=a_{0}+2 \sqrt{a_{-1} a_{1}} \cos \frac{\pi j}{n+1} \quad(j=1, \ldots, n) .
$$

Consequently, in our special case we have the eigenvalues

$$
\lambda_{j}\left(T_{n}(a)\right)=\cos \frac{\pi j}{n+1} \quad(j=1, \ldots, n),
$$

which implies that $\Lambda(a)=[-1,1]$.

Now let $\lambda \in(-1,1)$. There is a $y \in(0, \pi)$ such that $\lambda=\cos y$. The matrix $T_{n}(a-\lambda)$ has the eigenvalues

$$
\lambda_{j}\left(T_{n}(a-\lambda)\right)=\cos \frac{\pi j}{n+1}-\cos y \quad(j=1, \ldots, n) .
$$

Obviously,

$$
\left|\lambda_{j}\left(T_{n}(a-\lambda)\right)\right|=2\left|\sin \frac{1}{2}\left(\frac{\pi j}{n+1}-y\right) \sin \frac{1}{2}\left(\frac{\pi j}{n+1}+y\right)\right| \leq \pi\left|\frac{y}{\pi}-\frac{j}{n+1}\right| .
$$

Let

$$
\mu(n):=\min _{1 \leq j \leq n}\left|\lambda_{j}\left(T_{n}(a-\lambda)\right)\right| .
$$

We denote by $\varrho(\cdot)$ the spectral radius. Since

$$
\left\|T_{n}^{-1}(a-\lambda)\right\|_{p} \geq \varrho\left(T_{n}^{-1}(a-\lambda)\right)=1 / \mu(n),
$$

it suffices to prove that there is a $y \in(0, \pi)$ such that $\mu(n)>0$ for all $n \geq 1$ and

$$
\mu\left(n_{k}\right)<1 / \varphi\left(n_{k}\right)
$$

for infinitely many $n_{k}$. 
To find the desired $y$, we use a standard construction (see, e.g., [15, p. 6]). Pick any natural number $N_{1} \geq 1$ and choose natural numbers $N_{1}<N_{2}<N_{3}<\ldots$ successively by requiring that

$$
\pi \varphi\left(10^{N_{1}+\ldots+N_{k}}-1\right)<10^{N_{k+1}} \quad(k \geq 1) .
$$

Put

$$
n_{k}:=10^{N_{1}+\ldots+N_{k}}-1 \text { and } \frac{y}{\pi}:=10^{-N_{1}}+10^{-N_{1}-N_{2}}+10^{-N_{1}-N_{2}-N_{3}}+\ldots
$$

Obviously, the number $y / \pi$ is irrational. This implies that none of the eigenvalues (26) is zero, whence $\mu(n)>0$ for all $n \geq 1$. Since

$$
0<10^{-N_{1}}+10^{-N_{1}-N_{2}}+\ldots+10^{-N_{1}-\ldots-N_{k}}<1,
$$

it follows that

$$
10^{-N_{1}}+10^{-N_{1}-N_{2}}+\ldots+10^{-N_{1}-\ldots-N_{k}}=j_{k} 10^{-N_{1}-\ldots-N_{k}}
$$

with a natural number $1 \leq j_{k} \leq 10^{N_{1}+\ldots+N_{k}}-1=n_{k}$. We have

$$
\begin{aligned}
\frac{y}{\pi} & -\frac{j_{k}}{n_{k}+1} \\
& =\left(10^{-N_{1}}+10^{-N_{1}-N_{2}}+\ldots\right)-\left(10^{-N_{1}}+10^{-N_{1}-N_{2}}+\ldots+10^{-N_{1}-\ldots-N_{k}}\right) \\
& =10^{-N_{1}-N_{2}-\ldots-N_{k+1}}+10^{-N_{1}-N_{2}-\ldots-N_{k+2}}+\ldots,
\end{aligned}
$$

which shows that

$$
0<\frac{y}{\pi}-\frac{j_{k}}{n_{k}+1}<2 \cdot 10^{-N_{1}-N_{2}-\ldots-N_{k+1}}<10^{-N_{k+1}}<\frac{1}{\pi \varphi\left(n_{k}\right)},
$$

the last inequality resulting from (29). Thus, by (27),

$$
\mu(n) \leq\left|\lambda_{j_{k}}\left(T_{n}(a-\lambda)\right)\right|<\pi /\left(\pi \varphi\left(n_{k}\right)\right)=1 / \varphi\left(n_{k}\right) .
$$

This completes the proof of Theorem 1.5

The proof of Theorem 1.6 is the same as the one of Theorem 1.5. Indeed, if $a(z)=z+z^{-1}$, then the eigenvalues of $T_{n}(a)$ are (25), and hence we can repeat the above reasoning starting with (26).

7. Inside the essential spectrum. In this section we prove Theorems 1.7 to 1.9. The following result is Theorem 1.7 in analytic language.

THEOREM 7.1. Let a be a rational function without poles on $\mathbb{T}$ and let $\lambda \in a(\mathbb{T})$. Then $b(t):=a(t)-\lambda$ has zeros on $\mathbb{T}$. Suppose $b(t)$ has only one zero $t_{0} \in \mathbb{T}$ and

$$
b(t)=\left(t-t_{0}\right)^{\beta} t^{k} c(t), \quad t \in \mathbb{T}
$$

where $\beta \in \mathbb{N}, k \in \mathbb{Z}$, and $c$ is a rational function without poles and zeros on $\mathbb{T}$ whose winding number about the origin is zero. Then

$$
\kappa_{2}\left(T_{n}(a-\lambda)\right) \simeq n^{\beta} \quad \text { if } \quad-\beta \leq k \leq 0
$$


and there are constants $C>0$ and $\alpha>0$ such that

$$
\kappa_{2}\left(T_{n}(a-\lambda)\right) \geq C e^{\alpha n} \quad \text { if } \quad k<-\beta \quad \text { or } k>0 .
$$

Proof. Theorem 4.1(a) of [5] implies that $\kappa_{2}\left(T_{n}(b)\right) \geq C n^{\beta}$ in either case.

Suppose $-\beta \leq k \leq 0$. We then can write

$$
b(z)=\left(t-t_{0}\right)^{\beta-|k|}\left(1-\frac{t_{0}}{t}\right)^{|k|} c(t)
$$

with $\beta-|k| \geq 0$ and $|k| \geq 0$, and from Theorem 7.87(a) of [8] we deduce that

$$
\kappa_{2}\left(T_{n}(b)\right)=O\left(n^{\beta-|k|+|k|}\right)=O\left(n^{\beta}\right) .
$$

At this point the proof of (31) is complete.

Now suppose $k<-\beta$ or $k>0$. Since

$$
\widetilde{b}(t):=b(1 / t)=\left(1-t_{0} t\right)^{\beta} t^{-\beta-k} c(1 / t), \quad t \in \mathbb{T},
$$

and $\kappa_{2}\left(T_{n}(b)\right)=\kappa_{2}\left(T_{n}(\widetilde{b})\right)$, it suffices to consider the case $k>0$. Put $\chi(t)=(t-$ $\left.t_{0}\right)^{\beta} t^{k}$. Because the Fourier coefficients $\chi_{n}$ of $\chi$ vanish for $n \leq k-1$, we obtain as in Section 3 that

$$
\sigma_{k}\left(T_{n}(b)\right) \leq\|c\|_{W}\left\|A_{n} Q_{n-k}\right\|_{2}
$$

where

$$
A_{n}=T_{n}(\chi)+P_{n} H(\chi) H(\widetilde{c}) P_{n} T_{n}^{-1}(c) .
$$

As $T_{n}(\chi) Q_{n-k}=0$ and as $H(\chi)$ has only finitely many nonzero entries, we can also proceed as in Section 3 to conclude that

$$
\sigma_{k}\left(T_{n}(b)\right)=O\left(e^{-n \alpha}\right) \quad \text { as } n \rightarrow \infty,
$$

which gives (32).

We are now going to translate Theorem 7.1 into the language of Theorem 1.7.

Lемма 7.2. If $m$ is the winding number of $M_{\lambda}^{+}$, then $M_{\lambda}^{-}$has the winding number $m-1$.

Proof. Let $n$ be the winding number of $M_{\lambda}^{-}$. Fix a point $\mu \in M_{\lambda}^{+}$sufficiently close to $\lambda$. In a small neighborhood of $\lambda$, change $a(\mathbb{T})$ to a curve $\delta$ which first follows $\gamma_{\lambda}$, then goes into $M_{\lambda}^{+}$and encircles $\mu$ like a half-circle, and which finally goes back to $\gamma_{\lambda}$ and again follows $\gamma_{\lambda}$. Clearly,

$$
\text { wind }(\delta, \mu)=m-1 \text {. }
$$

On the other hand, since $\mu$ and $M_{\lambda}^{-}$are contained in the same connected component of $\mathbb{C} \backslash \delta$, we have

$$
\text { wind }(\delta, \mu)=n \text {. }
$$


Consequently, $n=m-1$.

Now let $r \in(0,1)$ and consider $b(r t)=a(r t)-\lambda$ for $t \in \mathbb{T}$.

Lemma 7.3. Let $r \in(0,1)$ be sufficiently close to 1 and suppose a point moves along the curve $a(r \mathbb{T})$. Then, in a small neighborhood of $\lambda$, this point is first in $M_{\lambda}^{+}$, then it encircles $\lambda$ exactly $[(\beta-1) / 2]$ times in the clockwise direction, after which the point is again in $M_{\lambda}^{+}$.

Proof. We have $a\left(t_{0}\right)=\lambda$ and

$$
a(z)=\lambda+\frac{1}{\beta !} a^{(\beta)}\left(t_{0}\right)\left(z-t_{0}\right)^{\beta}+O\left(\left(z-t_{0}\right)^{\beta+1}\right)
$$

with an analytic function $O\left(\left(z-t_{0}\right)^{\beta+1}\right)$ as $z \rightarrow t_{0}$. Thus, locally $a$ acts as the function

$$
z \mapsto \lambda+\frac{1}{\beta !} a^{(\beta)}\left(t_{0}\right)\left(z-t_{0}\right)^{\beta} .
$$

Let $U \subset \mathbb{C}$ and $V \subset \mathbb{C}$ be sufficiently small open neighborhoods of $t_{0}$ and 0 , respectively. Clearly, the images of $U \cap \mathbb{T}$ and $U \cap r \mathbb{T}$ under the map (33) have the same structure as the images of $V \cap C$ and $V \cap r C$ under the map $z \mapsto z^{\beta}$, where $C$ is a large circle in the upper half-plane that touches the real line at the origin. It can be checked straightforwardly that in the latter case the situation is as described in the lemma. प

Proof of Theorem 1.7. Write $b$ in the form (30). We then have

$$
b(r t)=\left(r t-t_{0}\right)^{\beta} r^{k} t^{k} c(r t), \quad t \in \mathbb{T} .
$$

Let $r \in(0,1)$ be sufficiently close to 1 . Then $\lambda \notin a(r \mathbb{T})$ and

$$
\begin{aligned}
& \text { wind }(a(r t), \lambda)=\operatorname{wind}(b(r t), 0) \\
& \quad=\operatorname{wind}\left(\left(r t-t_{0}\right)^{\beta}, 0\right)+k+\operatorname{wind}(c(r t), 0)=0+k+0=k .
\end{aligned}
$$

Evidently, $\max _{t \in \mathbb{T}}|a(r t)-a(t)| \rightarrow 0$ as $r \rightarrow 1-0$. This in conjunction with Lemma 7.3 shows that if $\mu \in M_{\lambda}^{-}$is sufficiently close to $\lambda$, then

$$
\text { wind }(a(r t), \lambda)=\operatorname{wind}(a(r t), \mu)=\operatorname{wind}(a(t), \mu)-\left[\frac{\beta-1}{2}\right] .
$$

From (34) and (35) we obtain

$$
k=\text { wind }(a(t), \mu)-\left[\frac{\beta-1}{2}\right] .
$$

By Lemma 7.2, wind $(a(t), \mu)=m-1$. Consequently,

$$
k=m-1-\left[\frac{\beta-1}{2}\right] .
$$


Since

$$
-\beta \leq m-1-\left[\frac{\beta-1}{2}\right] \leq 0 \quad \Longleftrightarrow \quad-\left[\frac{\beta}{2}\right] \leq m \leq\left[\frac{\beta+1}{2}\right],
$$

(7) and (8) follow from (31) and (32). This completes the proof of Theorem 1.7.

Proof of Theorem 1.8. If $\lambda \in a(\mathbb{T}) \backslash S(a)$ belongs to $\partial \operatorname{sp} T(a)$, then $M_{\lambda}^{+}$or $M_{\lambda}^{-}$ has the winding number zero. Thus, by Lemma $7.2, M_{\lambda}^{-}$has the winding number -1 or 0 . Since $[(\beta-1) / 2] \geq 0$ and $-[(\beta+2) / 2] \leq-1$ for every $\beta \in \mathbb{N}$, the assertion follows from Theorem 1.7. The proof of Theorem 1.8 is complete.

Proof of Theorem 1.9. The first part of the assertion is immediate from Theorem 1.8. So suppose $\lambda \in a(\mathbb{T}) \backslash(S(a) \cup \partial \operatorname{sp} T(a))$ and let $m$ be the winding number of $M_{\lambda}^{-}$. By Lemma 7.2, the winding number of $M_{\lambda}^{+}$is $m+1$. As $m \neq 0$ and $m+1 \neq 0$, we have $m \geq 1$ or $m \leq-2$. Theorem 1.7 with $\beta=1$ completes the proof of Theorem 1.9 .

EXAmPle 7.4. Let $a(t)=(t+1)^{3}$. We have

$$
a(\mathbb{T})=A_{1} \cup\{-1\} \cup A_{2} \cup\{0\},
$$

where

$$
\begin{aligned}
& A_{1}=\left\{a\left(e^{i \theta}\right):-2 \pi / 3<\theta<2 \pi / 3\right\}, \\
& A_{2}=\left\{a\left(e^{i \theta}\right): 2 \pi / 3<\theta<\pi \text { or }-\pi<\theta<-2 \pi / 3\right\} .
\end{aligned}
$$

Clearly, $S(a)=\{-1\}, \beta(\lambda)=1$ for $\lambda \in A_{1} \cup A_{2}$, and $\beta(\lambda)=3$ for $\lambda=0$. Theorem 1.7 implies that

$$
\kappa_{2}\left(T_{n}(a-\lambda)\right) \simeq \begin{cases}n & \text { for } \quad \lambda \in A_{1} \\ n^{3} & \text { for } \quad \lambda=0\end{cases}
$$

and that $\kappa_{2}\left(T_{n}(a-\lambda)\right)$ increases at least exponentially for $\lambda \in A_{2}$.

Theorems 7.1 and 1.7 are not applicable to the case $\lambda=-1$. We have

$$
a(t)+1=(t+1)^{3}+1=(t+2)(1-t \omega)\left(1-t \omega^{2}\right)
$$

with $\omega=-1 / 2+i \sqrt{3} / 2$. Put $\alpha=\omega$ and $\beta=\omega^{2}$. It is easily seen that

$$
T_{n}^{-1}(a+1)=T_{n}(c) T_{n}(\varphi)
$$

where

$$
c(t)=\frac{1}{2} \sum_{k=0}^{\infty}\left(-\frac{t}{2}\right)^{k}, \quad \varphi(t)=\sum_{k=0}^{\infty} \frac{\alpha^{k+1}-\beta^{k+1}}{\alpha-\beta} t^{k} .
$$

Clearly, $\left\|T_{n}(c)\right\|_{2} \simeq 1$. Since

$$
\left\|T_{n}(\varphi)\right\|_{1}=\left\|T_{n}(\varphi)\right\|_{\infty}=\sum_{k=0}^{n-1}\left|\frac{\alpha^{k+1}-\beta^{k+1}}{\alpha-\beta}\right| \simeq n,
$$


it follows that

$$
\left\|T_{n}(\varphi)\right\|_{2} \leq\left\|T_{n}(\varphi)\right\|_{1}^{1 / 2}\left\|T_{n}(\varphi)\right\|_{\infty}^{1 / 2}=O(n) .
$$

Considering the first column of of $T_{n}(\varphi)$, we see that actually $\left\|T_{n}(\varphi)\right\|_{2} \simeq n$.

EXAMPLE 7.5. There are $a$ such that $\kappa_{2}\left(T_{n}(a-\lambda)\right)$ does not grow at most polynomially although $\lambda \in \partial \operatorname{sp} T(a)$. Trivial examples are given by real-valued functions a. Here is a less trivial example. Let

$$
a(t)=t^{2}-t^{-2}-i\left(t-t^{-1}\right)
$$

Then

$$
a\left(e^{i \theta}\right)=e^{2 i \theta}-e^{-2 i \theta}-i\left(e^{i \theta}-e^{-i \theta}\right)=2 \sin \theta+2 i \sin 2 \theta
$$

Obviously, $0 \in \partial \operatorname{sp} T(a) \cap S(a)$. Since $T_{n}(a)$ is skew-symmetric and thus $D_{n}(a)=0$ if $n$ is odd, we see that $\kappa_{2}\left(T_{n}(a-0)\right)=\kappa_{2}\left(T_{n}(a)\right)=+\infty$ if $n$ is odd.

We finally consider the simplest case of points in $S(a) \cap \partial \operatorname{sp} T(a)$ : we assume that $b(t)=a(t)-\lambda$ has exactly two simple and distinct zeros on $\mathbb{T}$ and that, in a sufficiently small neighborhood of $\lambda$ and after appropriate translation, rotation, and straightening, $a(\mathbb{T})$ looks like the union of the positively oriented $x$-axis and the positively oriented $y$-axis in a neighborhood of the origin of the $x, y$-plane. The winding numbers of the four connected components of $\mathbb{C} \backslash a(\mathbb{T})$ which touch $\lambda$ associate four integers $m_{++}$, $m_{-+}, m_{--}, m_{+-}$with the quadrants of the $x, y$-plane. Since $\lambda \in \partial \operatorname{sp} T(a)$, at least one of the four numbers $m_{ \pm, \pm}$must be zero. Taking into account Lemma 7.2 , we see that there are exactly three cases:

case $1: m_{++}=0, m_{-+}=1, m_{--}=0, m_{+-}=-1$;

case $2: m_{++}=-1, m_{-+}=0, m_{--}=-1, m_{+-}=-2$;

case 3: $m_{++}=1, m_{-+}=2, m_{--}=1, m_{+-}=0$.

In Example 7.5 we encountered case 1 and observed that $\kappa_{2}\left(T_{n}(a-\lambda)\right)$ need not grow at most polynomially in this case.

THEOREM 7.6. In cases 2 and 3 , the condition numbers $\kappa_{2}\left(T_{n}(a-\lambda)\right)$ grow at most polynomially.

Proof. Let us consider case 3, the other case can be reduced to this case by passage to adjoints. We have

$$
b(t)=a(t)-\lambda=(t-\alpha)(t-\beta) c(t), \quad t \in \mathbb{T},
$$

where $\alpha \in \mathbb{T}, \beta \in \mathbb{T}, \alpha \neq \beta$, and $c$ has neither poles nor zeros on $\mathbb{T}$. Clearly,

$$
\text { wind }(c(t), 0)=\lim _{r \rightarrow 1-0} \text { wind }(b(r t), 0)=\lim _{r \rightarrow 1-0} \text { wind }(a(r t), \lambda) \text {. }
$$

From Lemma 7.3 we deduce that wind $(a(r t), \lambda)=0$ if $r \in(0,1)$ is sufficiently close to 1 . Hence, wind $(c, 0)=0$. From [7, Lemma 3 and Theorem 1] we therefore obtain that

$$
\left\|T_{n}^{-1}(b)\right\|_{\infty}=O\left(n^{2 \cdot 1-1+\varepsilon}\right)=O\left(n^{1+\varepsilon}\right)
$$


where $\varepsilon>0$ can be chosen abitrarily. Since

$$
\left\|T_{n}^{-1}(b)\right\|_{2} \leq \sqrt{n}\left\|T_{n}^{-1}(b)\right\|_{\infty},
$$

it results that $\kappa_{2}\left(T_{n}(a-\lambda)\right)=O\left(n^{3 / 2+\varepsilon}\right)$.

Notice that in Example 7.4 we have case 3 at $\lambda=-1$.

Appendix. In connection with Theorem 1.6, it is rather instructive to consider $\kappa_{2}\left(T_{n}(a-\lambda)\right)$ for $a(z)=z+z^{-1}$ and some selected values $\lambda \in \Lambda(a)=[-2,2]$. We first note that

$$
\left\|T_{n}(a-\lambda)\right\|_{2} \rightarrow\|a-\lambda\|_{\infty}=\max _{0 \leq \theta \leq 2 \pi}|2 \cos \theta-\lambda| .
$$

Since $T_{n}(a-\lambda)$ is Hermitian, we have

$$
\left\|T_{n}^{-1}(a-\lambda)\right\|_{2}=\varrho\left(T_{n}^{-1}(a-\lambda)\right)=1 / \mu(n),
$$

where $\mu(n)$ is given by (28). From (36) and (37) we get

$$
\kappa_{2}\left(T_{n}(a-\lambda)\right) \sim \frac{\|a-\lambda\|_{\infty}}{\mu(n)} .
$$

Suppose first that $\lambda=2$. Then

$$
\mu(n)=\min _{1 \leq j \leq n}\left|2 \cos \frac{\pi j}{n+1}-2\right|=2-2 \cos \frac{\pi}{n+1} \sim \frac{\pi^{2}}{n^{2}},
$$

and since $\|a-2\|_{\infty}=4$, we deduce from (38) that

$$
\kappa_{2}\left(T_{n}(a-2)\right) \sim 4 \pi^{2} / n^{2} .
$$

Analogously,

$$
\kappa_{2}\left(T_{n}(a+2)\right) \sim 4 \pi^{2} / n^{2} .
$$

We remark that the last two relations are already in Grenander and Szegö's book [13].

Now let $\lambda=2 \cos y$ with $y \in(0, \pi)$. We have

$$
\left|2 \cos \frac{\pi j}{n+1}-2 \cos y\right|=4\left|\sin \frac{\pi}{2}\left(\frac{j}{n+1}-\frac{y}{\pi}\right)\right|\left|\sin \frac{\pi}{2}\left(\frac{j}{n+1}+\frac{y}{\pi}\right)\right| .
$$

Suppose $y / \pi=l / m$ is rational. Clearly, we can assume that $m \geq 2$. In the case where $n+1 \equiv 0(\bmod m)$, we have $\mu(n)=0$. Hence,

$$
\kappa_{2}\left(T_{n}(a-\lambda)\right)=\infty \quad \text { if } \quad n+1 \equiv 0(\bmod m) .
$$

If $n+1 \not \equiv 0(\bmod m)$, there is a unique $k \in\{1,2, \ldots,[m / 2]\}$ such that $l(n+1) \equiv$ $\pm k(\bmod m)$. A little thought reveals that

$$
\mu(n) \sim 4 \frac{\pi}{2} \frac{k}{m(n+1)} \sin \frac{\pi}{2}\left(\frac{l}{m}+\frac{l}{m}\right) \sim \frac{2 \pi k}{m}\left(\sin \frac{\pi l}{m}\right) \frac{1}{n},
$$


whence

$$
\kappa_{2}\left(T_{n}(a-\lambda)\right) \sim \frac{\|a-\lambda\|_{\infty}}{2 \pi k} \frac{m}{\sin (\pi l / m)} n .
$$

Consequently, the sequence $\left\{\kappa_{2}\left(T_{n}(a-\lambda)\right)\right\}_{n=1}^{\infty}$ can be divided into the subsequence $\{\infty, \infty, \ldots\}$ and a finite number of subsequences $\left\{\kappa_{2}\left(T_{n_{i}^{(k)}}(a-\lambda)\right)\right\}$ for which

$$
\kappa_{2}\left(T_{n_{i}^{(k)}}(a-\lambda)\right) \sim C_{k} n_{i}^{(k)}
$$

with specific constants $C_{k}$. In particular,

$$
\begin{aligned}
& \kappa_{2}\left(T_{n}(a-\lambda)\right)=\infty \text { for } n \equiv-1(\bmod m), \\
& \kappa_{2}\left(T_{n}(a-\lambda)\right) \simeq n \text { for } n \not \equiv-1(\bmod m) .
\end{aligned}
$$

Finally, suppose $y / \pi$ is irrational. Then, by (39),

$$
\kappa_{2}\left(T_{n}(a-\lambda)\right)<\infty \text { for all } n \geq 1
$$

If $y / \pi$ is algebraic of degree $\beta(\geq 2)$, then Liouville's theorem ensures the existence of a constant $c>0$ such that

$$
\left|\frac{y}{\pi}-\frac{j}{n+1}\right| \geq \frac{c}{(n+1)^{\beta}}
$$

for all $n \geq 1$. Thus, in this "tame" case we still have the estimate

$$
\kappa_{2}\left(T_{n}(a-\lambda)\right) \leq C n^{\beta} \quad \text { for all } \quad n \geq 1
$$

with some constant $C<\infty$ independent of $n$. The derivation of (40) from Liouville's theorem can also be found in Berg's book [1, pp. 105-106].

By a theorem of Dirichlet (see, e.g., [15, p. 4]), for every (not necessarily algebraic) irrational number $y / \pi$ there exists a sequence $n_{1}<n_{2}<n_{3}<\ldots$ of natural numbers such that the inequality

$$
0<\left|\frac{y}{\pi}-\frac{j_{k}}{n_{k}+1}\right|<\frac{1}{\left(n_{k}+1\right)^{2}}
$$

has a solution $j_{k} \in\left\{1,2, \ldots, n_{k}\right\}$. This implies that

$$
\kappa_{2}\left(T_{n_{k}}(a-\lambda)\right) \geq D n_{k}^{2}
$$

with some constant $D \in(0, \infty)$ which does not depend on $n_{k}$.

Fazit: if $y / \pi$ is irrational, there is always a subsequence $\left\{n_{k}\right\}$ such that (41) holds; for algebraic $y / \pi$ we nevertheless have (40), but for transcendental $y / \pi$ the situation may be as "wild" as described by Theorem 1.6. 


\section{REFERENCES}

[1] L. Berg, Lineare Gleichungssysteme mit Bandstruktur, Deutscher Verlag der Wissenschaften, Berlin 1986.

[2] R. Bhatia, Matrix Analysis, Springer-Verlag, New York 1996.

[3] A. Böttcher, Status report on rationally generated block Toeplitz and Wiener-Hopf determinants, Unpublished manuscript, 34 pages, 1989 (available from the author on request).

[4] A. Böttcher, On the approximation numbers of large Toeplitz matrices, Doc. Math., 2:1-29, 1997.

[5] A. Böttcher and S. Grudsky, On the condition numbers of large semi-definite Toeplitz matrices, Linear Algebra Appl., 279:285-301, 1998.

[6] A. Böttcher, S. Grudsky, and B. Silbermann, Norms of inverses, spectra, and pseudospectra of large truncated Wiener-Hopf operators and Toeplitz matrices, New York J. Math., 3:1-31, 1997.

[7] A. Böttcher and B. Silbermann, The asymptotic behavior of Toeplitz determinants for generating functions with zeros of integral orders, Math. Nachr., 102: 79-105, 1981.

[8] A. Böttcher and B. Silbermann, Analysis of Toeplitz Operators, Akademie-Verlag, Berlin 1989 and Springer Verlag, Berlin, Heidelberg, New York 1990.

[9] A. Böttcher and B. Silbermann, Introduction to Large Truncated Toeplitz Matrices, SpringerVerlag, New York 1998.

[10] K.M. Day, Toeplitz matrices generated by the Laurent series expansion of an arbitrary rational function, Trans. Amer. Math. Soc., 206:224-245, 1975.

[11] K.M. Day, Measures associated with Toeplitz matrices generated by the Laurent expansion of rational functions, Trans. Amer. Math. Soc., 209:175-183, 1975.

[12] I. Gohberg and I.A. Feldman, Convolution Equations and Projection Methods for Their Solution, Amer. Math. Soc., Providence, RI, 1974 [Russian original: Nauka, Moscow 1971].

[13] U. Grenander and G. Szegö, Toeplitz Forms and Their Applications, University of California Press, Berkeley and Los Angeles 1958.

[14] I.I. Hirschman, Jr., The spectra of certain Toeplitz matrices, Illinois J. Math., 11:145-159, 1967.

[15] E. Hlawka, J. Schoissengaier, and R. Taschner, Geometric and Analytic Number Theory, Springer-Verlag, Berlin, Heidelberg, New York 1991.

[16] M. Kac, W.L. Murdock, and G. Szegö, On the eigenvalues of certain Hermitian forms, $J$. Rational Mech. Anal., 2:767-800, 1953.

[17] S.V. Parter, Extreme eigenvalues of Toeplitz forms and applications to elliptic difference equations, Trans. Amer. Math. Soc., 99:153-192, 1966.

[18] S. Roch and B. Silbermann, Index calculus for approximation methods and singular value decomposition, J. Math. Anal. Appl., 225:401-426, 1998.

[19] M. Rosenblatt, Some purely deterministic processes, J. Math. Mech., 6:801-810, 1957.

[20] P. Schmidt and P. Spitzer, The Toeplitz matrices of an arbitrary Laurent polynomial, Math. Scand., 8:15-38, 1960.

[21] S. Serra, On the extreme eigenvalues of Hermitian (block) Toeplitz matrices, Linear Algebra Appl., 270:109-129, 1997.

[22] S. Serra, How bad can positive definite Toeplitz matrices be?, Talk given at the conference "Fourier Analysis and Applications", Kuwait University, May 1998.

[23] H. Widom, On the eigenvalues of certain Hermitian operators, Trans. Amer. Math. Soc., 88:491-522, 1958. 